\title{
A Genetic Strategy to Eliminate Self-Activator Baits Prior to High-Throughput Yeast Two-Hybrid Screens
}

\author{
Albertha J.M. Walhout and Marc Vidal ${ }^{1}$ \\ Massachusetts General Hospital Cancer Center, Charlestown, Massachusetts 02129 USA
}

\begin{abstract}
Large-scale sequencing projects have predicted high numbers of gene products for which no functional information is yet available. Hence, large-scale projects, such as gene knockouts, gene expression profiles, and protein-interaction mapping, are currently under way to initiate the understanding of the function of these gene products. The high-throughput strategies that are currently being developed to generate protein-interaction maps include automated versions of the yeast two-hybrid system. These strategies rely on the large-scale construction of DNA-binding domain/protein-of-interest hybrid constructs (DB-X baits). An inherent problem of large-scale two-hybrid systems is that a high percentage of cloned sequences encode polypeptides that, when fused to $\mathrm{DB}$, can activate transcription in the absence of any two-hybrid-interacting partner protein. Here, we describe and validate a genetic strategy that efficiently eliminates such self-activator baits prior to screening procedures. The strategy is based on a negative-growth selection and is compatible with high-throughput settings.
\end{abstract}

Complete genome sequences are available for several model organisms [Escherichia coli (Blattner et al. 1997), Saccaharomyces cerevisiae (Goffeau et al. 1997), and Caenorhabditis elegans (C. elegans sequencing consortium 1998)] and pathogens (e.g., see Stephens et al. 1998). These genome sequences have revealed the need for large-scale projects to convert genomic information into functional information (Guyer and Collins 1995; Lander 1996; Fields 1997). For example, in >30 years, conventional biology has assigned a function to $\sim 500$ C. elegans genes of the 19,099 predicted ORFs (e.g., see Walhout et al. 1998). Thus, $>97 \%$ of the C. elegans genome remains to be characterized functionally.

Several laboratories have initiated the development of genome-wide functional analysis projects to help address the function of genes identified by largescale sequencing efforts. Such projects, collectively referred to as functional genomics, are expected to predict gene functions and lead to hypotheses at a drastically increased rate. Functional genomics projects include genome-wide expression analysis (Schena et al. 1995), large-scale gene knockouts (e.g., see Smith et al. 1996), and protein-interaction maps (Evangelista et al. 1997). Protein-interaction maps can be envisioned as publicly available databases containing large numbers of pairs of potentially interacting proteins (Walhout et al. 1998). Their usefulness stems from the fact that many biological processes rely heavily on proteinprotein interactions, from the formation of large enzymatic protein complexes (e.g., see Koleske and Young

'Corresponding author.

E-MAIL: Vidal@helix.mgh.harvard.edu; FAX (617) 726-7808.
1994) to the regulatory roles of signal-transduction pathways (e.g., see Choi et al. 1994). So far, most strategies proposed to generate comprehensive proteininteraction maps have relied on high-throughput versions of the yeast two-hybrid system (Bartel et al. 1996; Fromont-Racine et al. 1997; Flores et al. 1999; A.J.M. Walhout, R. Sordella, X. Lu, J. Hartley, G. Temple, M. Brasch, N. Thierry-Mieg, and M. Vidal, in prep.).

The yeast two-hybrid system is a powerful genetic method to identify potential protein-protein interactions. This system circumvents tedious and timeconsuming biochemical methods and is thus amenable to automated high-throughput settings. The twohybrid system makes use of the observation that transcription factors are generally composed of two separable domains, a sequence-specific DNA-binding domain (DB) and a transactivation domain (AD). When a protein $\mathrm{X}$ is fused to $\mathrm{DB}(\mathrm{DB}-\mathrm{X})$ and a protein $\mathrm{Y}$ to $\mathrm{AD}$ (AD-Y), an interaction between $\mathrm{X}$ and $\mathrm{Y}$ can be detected by the reconstitution of a functional transcription factor (DB-X/AD-Y) (Fields and Song 1989). In the most common application, $\mathrm{X}$ is a single protein, usually referred to as the bait. To identify potential interaction partners of $\mathrm{X}$, the $\mathrm{DB}-\mathrm{X}$ bait is screened against a complex genomic or cDNA AD-Y library.

There is a major limitation inherent to the twohybrid system. DB-X fusions that can activate transcription independently of an interaction with an $\mathrm{AD}-\mathrm{Y}$ protein (self activators) cannot be used in conventional forward two-hybrid screens. Self activators include proteins that act as transcriptional activators in their respective organisms and maintain this ability in yeast (e.g., see Du et al. 1996). They also include proteins that normally act in other processes but ex- 
hibit transcriptional activity when tethered to a promoter in yeast cells (e.g., see Hu et al. 1997). In highthroughput settings, large numbers of baits need to be generated and screened against AD-Y libraries. This can be accomplished by use of either one of two different strategies. In one strategy, referred to here as the random strategy, a complex library of randomly generated DB-X genomic or cDNA clones is screened against an AD-Y library by yeast-mating procedures (Bartel et al. 1996; Vidal 1997) This approach has been used previously to generate an interaction map for the 55 proteins encoded by the bacteriophage T7 genome (Bartel et al. 1996). A more directed strategy has also been proposed in which large numbers of ORFs are PCR amplified and individually cloned into DB plasmids (Hudson et al. 1997; Walhout et al. 1998). Here again, mating can be used to screen a set of predefined DB-X fusions against an AD-Y library (Finley and Brent 1994; Fromont-Racine et al. 1997).

In addition to the two classes of self activators described above, both high-throughput strategies are complicated by the occurrence of spurious DB-X self activators originating from cloning artifacts. These can include genomic or cDNA fragments cloned out-offrame in the random strategy. For example, $\sim 1 \%$ of randomly generated DNA sequences from E. coli encode polypeptides that can activate transcription in yeast when fused to DB (Ma and Ptashne 1987). In the directed strategy, spurious self activators originating from PCR-induced mutations can also be a problem if the bait-encoding plasmids are not systematically verified by sequencing (see below).

The percentage of spurious self activators in particular pools of DB-X baits can be as high as 5\% $\left(\sim 5 \times 10^{-2}\right)$ (see below), whereas the frequency of potential interactors for a single bait is usually $<50 / 10^{6}$ $\left(\sim 5 \times 10^{-5}\right)$ in non-normalized cDNA libraries. Because the frequency of self activators can be higher than the frequency of genuine interactors by several orders of magnitude, it is crucial for the quality of protein-interaction maps to develop a convenient method to efficiently eliminate self activators.

Here, we describe such a method, which is applicable to both random and directed strategies prior to high-throughput two-hybrid screens.

\section{RESULTS AND DISCUSSION}

\section{A Genetic Selection to Eliminate Self-Activator Baits}

Most versions of the yeast two-hybrid system rely on genetic selections on the basis of the transcriptional activation of reporter genes required for growth (e.g., see Vidal and Legrain 1999). When activated by DB-X/ $\mathrm{AD}-\mathrm{Y}$ interactions, these reporter genes allow cell growth under specific conditions (positive selections). Under such conditions, cells expressing DB-X self acti- vators are equally able to grow and form colonies in the absence of an AD-Y interaction partner. Such a positive growth phenotype can be used to identify and manually remove self activators from a pool of yeast colonies (Bartel et al. 1996). However, this requires time-consuming manipulations and is therefore not applicable to large-scale projects.

Recently, we have developed a reverse two-hybrid system, in which the transcriptional activation of a counterselectable reporter gene is disadvantageous for growth (Vidal et al. 1996a). When activated by DB-X/ $\mathrm{AD}-\mathrm{Y}$ interactions, this reporter gene prevents growth under particular conditions (negative selection). The reverse two-hybrid system is most commonly used for the selection of interaction-defective alleles or dissociator peptides or compounds (Vidal et al. 1996b; Vidal 1997; Vidal and Endoh 1999). These reagents can then be used to validate potential interactions obtained by forward two-hybrid selections. Here, we make use of such a negative selection to eliminate self activators from large pools of yeast cells. Because it is based on a genetic selection, this approach does not require timeconsuming manipulations and is thus applicable to large-scale projects.

Our version of the two-hybrid system utilizes two selectable markers that can be transcriptionally activated by two-hybrid interactions (Vidal 1997). The marker used for positive selection is the GAL1::HIS3 reporter gene (Durfee et al. 1993). The HIS3 gene encodes an enzyme involved in histidine biosynthesis that can be specifically inactivated by the competitive inhibitor 3-aminotriazole (3AT). Hence, it is possible to titrate the level of 3AT so that growth of yeast cells that express only basal levels of HIS3 would be prevented. Under these conditions, even moderate increases of HIS3 expression confer a growth advantage.

The counter-selectable reporter gene used for negative selection is SPAL10::URA3 (Vidal et al. 1996a). The URA3 gene encodes an enzyme required for uracil biosynthesis and can be used for positive growth selections on medium lacking uracil. However, this enzyme can also convert the nontoxic 5-fluoorotic acid (5FOA) into a toxic byproduct (Boeke et al. 1984). Thus, on medium containing both uracil and 5FOA, expression of URA3 is toxic.

To examine whether negative selection with 5FOA can be used for the elimination of self activators, we asked the following two questions. Can self activators be efficiently eliminated with 5FOA? And, how successful are subsequent two-hybrid selections? We addressed these questions in the context of both the random and the directed strategies described above.

\section{General Scheme}

The rationale behind our experiments is analogous to any preclearing step of experiments in which back- 
ground activity needs to be reduced or removed prior to performing the relevant functional test(s). In our scheme (Fig. 1), haploid yeast cells of one mating type are first transformed with a pool of DB-X bait plasmids. As described above, these might include a subpopulation of molecules encoding natural or spurious self activators. Transformants are selected on plates lacking leucine (Sc-Leu) because, in our system, the DB plasmid contains the LEU2 selectable marker. The Leu ${ }^{+}$ transformants are then transferred to the 5FOA negative-selection plates by replica plating. As described above, self-activator-expressing colonies should not be able to grow under these conditions. After a two-day incubation, the 5FOA resistant colonies are transferred to fresh Sc-Leu plates for recovery. Subsequently, the non-self-activator colonies can be mated with cells of the opposite mating type containing an AD-Y library in a TRP1 vector. Diploids are selected on plates lacking both leucine and tryptophan (Sc-Leu-Trp) and can be submitted to the 3AT-positive selection for the identification of DB-X/AD-Y interactions.

To control for the efficiency of the preclearing step, the haploid colonies expressing DB-X baits are also transferred to 3AT-positive selection plates, both prior (plate 3AT\#1) and subsequent (plate 3AT\#2) to the preclearing step (Fig. 1). When colonies are still able to grow on the $3 \mathrm{AT} \# 2$ plate, additional preclearing steps can be performed (Fig. 1, dotted arrow).

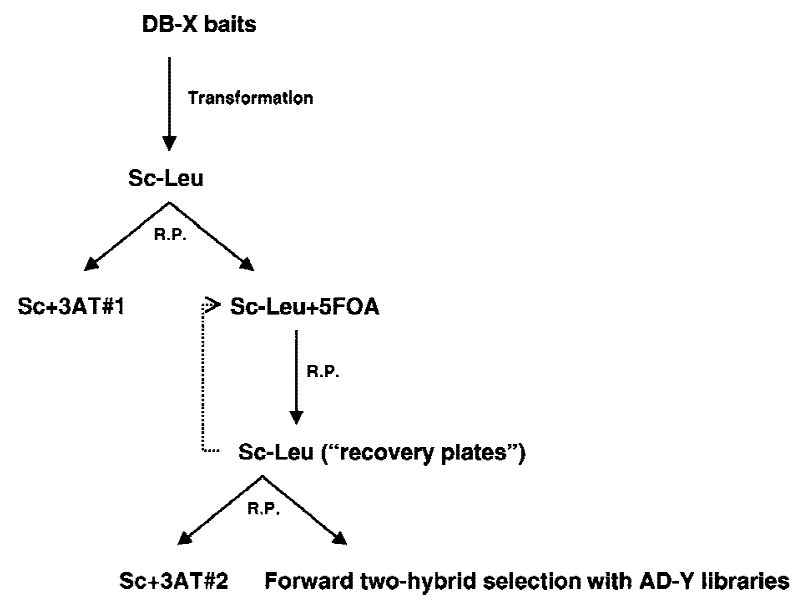

Figure 1 Flowchart of the self-activator preclearing step followed by forward two-hybrid screens. DB-X bait constructs are transformed and selected on synthetic plates lacking leucine (ScLeu). These plates are replica-plated (R.P.) onto two plates. One plate contains $20 \mathrm{~mm}$ 3AT ( $\mathrm{Sc}+3 \mathrm{AT} \# 1$ ) and is used to monitor the number of self activators prior to the preclearing step; the other contains 5FOA (0.2\%) (SC-Leu+5FOA) and is used to eliminate self activators. The Sc-Leu+5FOA plates are then replica plated onto Sc-Leu plates for recovery. The recovery plates are transferred to a second $\mathrm{Sc}+3 \mathrm{AT}$ plate $(20 \mathrm{~mm})(\mathrm{Sc}+3 \mathrm{ATH} 2)$ to assess the efficiency of self-activator elimination. If necessary, the whole procedure can be repeated. After efficient elimination of self activators, the plates can be used for forward two-hybrid screens. Representatives of the three plates indicated in red in this scheme are shown in the next two figures.

\section{Elimination of Self Activators from a DB-X cDNA Library}

To test the scheme described above in the context of the random strategy, we used two non-normalized mouse 13.5-day embryonic cDNA libraries, one fused to DB (DB-cDNA) and the other to AD (AD-cDNA). Different versions of both libraries have already been used to identify a large number of valid interactors (Chevray and Nathans 1992; Hu et al. 1997). We reasoned that the high complexity of these mammalian cDNA libraries would provide an ideal test to evaluate the efficiency of the self-activator preclearing step. In addition, we reasoned that non-normalized libraries provide a good test for the efficiency of recovering protein-protein interactions in subsequent forward two-hybrid selections because several cDNAs that are known to be highly represented in embryonic libraries encode proteins that physically interact (Soares et al. 1994; see below).

The DB-cDNA library was transformed into MATa cells to obtain $\sim 50,000$ transformants. These were replica plated onto both $3 \mathrm{AT}$ and 5FOA plates. As seen in Figure 2A, 2500 colonies were resistant to 3AT and are thus presumed to express self-activator fusions. This number corresponds to $\sim 5 \%$ of the mouse cDNAs fused to DB and is consistent with the data reported previously (Ma and Ptashne 1987).

To estimate the efficiency of the preclearing step, the recovery plates were also replica plated onto 3AT plates (Figs. 1 and 2A). The preclearing of the DB-cDNA library was compared with that of a reference that consists of a well-described self-activator construct [DBDCC (Hu et al. 1997)] diluted 100-fold in a solution of empty DB plasmid. Preclearing conditions have already been optimized to allow efficient elimination of this DB-DCC control self activator (Vidal 1997). In the experiment shown here, a few hundred colonies had survived the first negative selection and thus the 5FOA-negative selection was repeated (not shown). As seen in Figure 2A (right, bottom), no 3AT resistant colonies could be detected after two rounds of 5FOA selections, indicating that self activators had been eliminated efficiently.

Simultaneously, the AD-cDNA library was transformed into MAT $\alpha$ cells to obtain $\sim 50,000$ transformants. No growing colonies were obtained when the corresponding $\operatorname{Trp}^{+}$transformants were replica plated onto 3AT plates, indicating that the frequency of $\mathrm{AD}-\mathrm{Y}$ self activators is much lower than that of DB-X self activators $(<1 / 50,000)$ (data not shown). This result was expected, because in the absence of a sequencespecific DNA-binding domain, such as DB, the fusion protein is not tethered to the reporter genes and therefore does not activate transcription.

To demonstrate that forward two-hybrid selections can be applied after self-activator preclearing, 
A

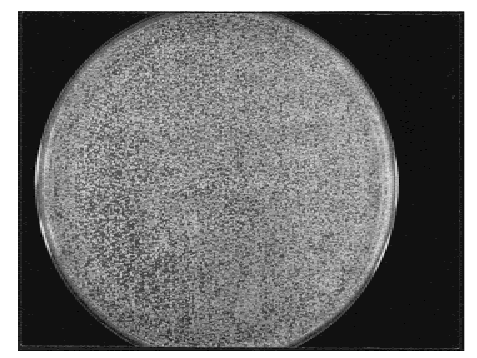

Sc-Leu

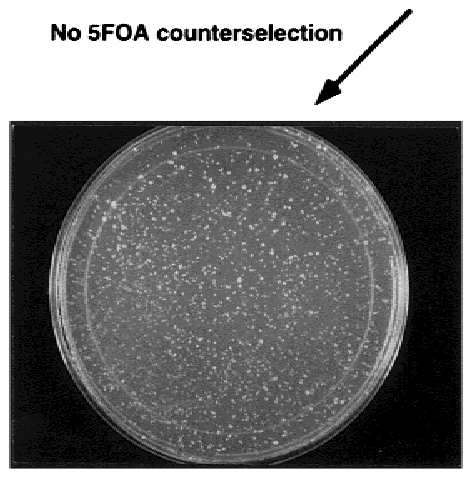

$\mathrm{Sc}+3 \mathrm{AT} \# 1$

B

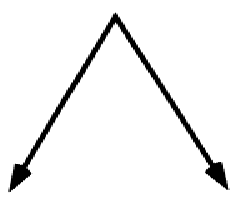

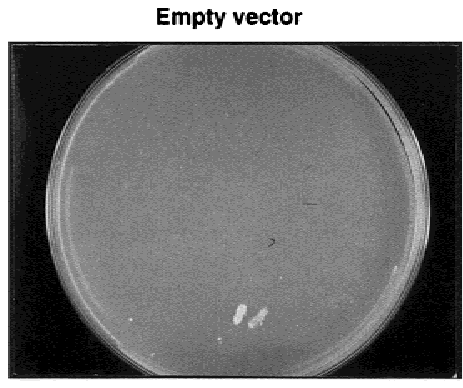

Sc+3AT

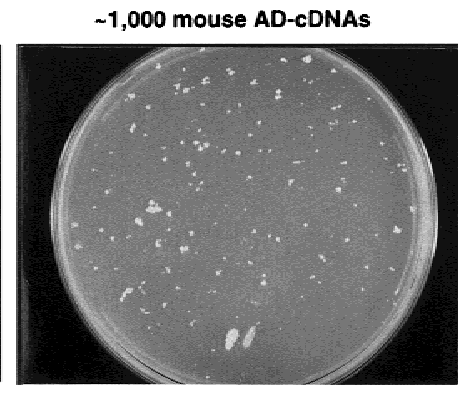

Sc+3AT
Figure 2 Preclearing of self-activator baits from random DB-CDNA libraries. $(A)$ The mouse 13.5-day embryonic DB-CDNA library cloned in a LEU2 plasmid was transformed into MaV103 cells. The $\sim 50,000$ resulting $\mathrm{Leu}^{+}$transformants were treated as described in the legend to Fig. 1. The plates shown here correspond to the Sc-Leu, Sc+3AT\#1, and Sc+3AT\#2 plates indicated in red in Fig. 1. The Sc-3AT plate at right contains two control patches. (B) A mouse 13.5-day embryonic AD-cDNA library cloned in a TRP1 plasmid was transformed into MaV203 cells and the resulting transformants were mated with the precleared DB-CDNA transformants shown in $A$. The plate at right shows the resulting 3AT-growing colonies indicative of potential two-hybrid interactions. As a negative control, MaV203 was transformed with a TRP1 empty vector and mated with the precleared DB-cDNA transformants (left). Both plates contain two control patches at the bottom.

empty AD plasmid alone were used. As expected from the efficiency of preclearing (Fig. 2A), diploids originating from this mating experiment produced only a few 3AT-resistant colonies (Fig. 2B, left). In contrast, when $\mathrm{AD}-\mathrm{Y}$ and precleared $\mathrm{DB}-\mathrm{X}$ cells were mated, a substantial number $(\sim 200)$ of 3AT-resistant colonies was observed (Fig. $2 \mathrm{~B}$, right). These colonies also exhibited uracil prototrophy and $\beta$-galactosidase activity (Vidal 1997), indicating that they represent genuine two-hybrid interactors (data not shown).

Next, the identity of the two potential interacting proteins from a dozen 3ATresistant colonies was determined by sequencing DB-X and AD-Y PCR products amplified directly from yeast colonies (Wang et al. 1996). The majority of interactor pairs consisted of $\alpha$ - and $\beta$-globin, either in the DB- $\alpha$-globin/AD- $\beta$-globin or in the DB- $\beta$-globin/AD- $\alpha$-globin orientation. To evaluate the relative presence of the $\alpha$-globin/ $\beta$-globin interaction in a larger number of 3AT-growing colonies, a yeastcolony hybridization experiment with $\alpha$-globin or $\beta$-globin radiolabeled DNA probes was performed. Approximately 95\% of the 3AT-growing colonies represented the globin-interaction pair, which can be explained by the relative abundance of $\alpha$ and $\beta$-globin cDNAs in both the DB-X and the AD-Y libraries ( 3\%) (data not shown).

The recovery of the $\alpha$-globin/ $\beta$-globin interaction in this experiment demonstrates that relevant interactions can be identified after the elimination of selfactivator baits by use of 5FOA. In addition, it indicates that the application of random two-hybrid screens in high-throughput settings requires the use of normalized libraries (Soares et al. 1994).

\section{Elimination of Mutant Self- \\ Activators Generated by PCR}

Although potentially powerful, the random strategy for generating proteininteraction maps described above is limited by the fact that it is not directly connected to any particular biological question.

Therefore, several laboratories have re-

1000 MAT $\alpha$ AD-Y transformants were pooled, plated, and subsequently replica plated onto $\sim 50,000$ precleared MATa DB-X colonies to allow mating (Fig. 2A). The resulting diploids were selected on Sc-Leu-Trp. As a negative control, MAT $\alpha$ cells transformed with the cently embarked in a more directed approach involving large numbers of defined $\mathrm{DB}-\mathrm{X}$ bait constructs rather than random DB-X cDNA libraries. Usually, the baits are selected on the basis of their known or suspected involvement in a biological process of interest. 
For example, a protein-interaction map of yeast proteins involved in RNA splicing has been described recently (Fromont-Racine et al. 1997). In the directed strategy, sets of predefined ORFs must be inserted one-by-one into the DB plasmid prior to performing two-hybrid screens.

Conventional cloning methods can be time consuming and are thus not compatible with high-throughput settings. Therefore, they are usually replaced by the PCR-Gap repair transformation method (e.g., see Vidal 1997). This method allows the rapid cloning of PCR products into vectors such as the DB plasmid by direct recombination in yeast. ORFs are amplified by PCR with primers containing, at their 3 'end, the genespecific sequences needed for amplification, and at their 5'end, a tail sequence identical to the polylinker sequence of the DB plasmid. The tail sequences are used to promote recombination of the PCR products into the DB plasmids in vivo. The Gap-repair method can easily be automated, because each step of the protocol can be performed in 96-well plates.

Our laboratory is involved in a C. elegans protein-interaction mapping project with the directed strategy (Walhout et al. 1998; A.J.M. Walhout, R. Sordella, X. Lu, J. Hartley, G. Temple, M. Brasch, N. Thierry-Mieg, and M. Vidal, in prep). In general, large numbers of worm ORFs are amplified by PCR and inserted into the DB plasmid by Gap repair. In the course of these experiments, we noticed the occurrence of an unexpected class of self activators that could greatly complicate the outcome of the directed strategy, even when applied to other genomes. For several baits tested in our laboratory so far, a small, but significant, fraction of the transformants ( $5 \%)$ exhibited growth on 3-AT plates. According to our preliminary observations, the number of baits belonging to this class might be as high as $15 \%$. These self activators are derived from PCR-induced mutations as shown below and thus we refer to them as spurious self-activators. They represent false-positive clones and thus need to be removed prior to applying two-hybrid selections.

The C. elegans lin-5 gene (M. Lorson, H.R. Horvitz, and S. van den Heuvel, in prep.) is used here to exemplify the preclearing procedure for such spurious selfactivator baits (Fig. 3). The lin-5 ORF was amplified by PCR with a CMV-lin-5 plasmid as template DNA and primers that contain both lin-5- and vector-specific sequences (see Vidal 1997). The lin-5 PCR fragment was transformed into MaV103 yeast cells along with linearized DB vector and several hundreds of transformants were selected on Sc-Leu plates. Approximately 5\% of the $\mathrm{Leu}^{+}$transformants could confer a 3AT-resistant
A

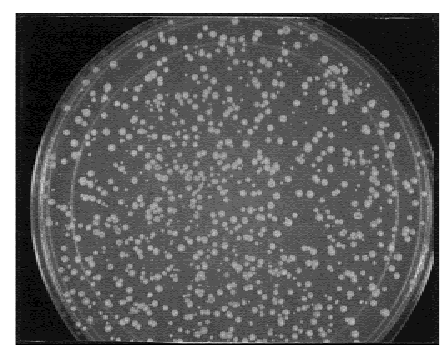

DB-LIN-5

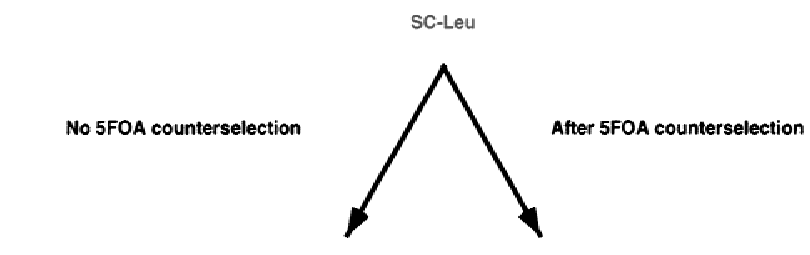

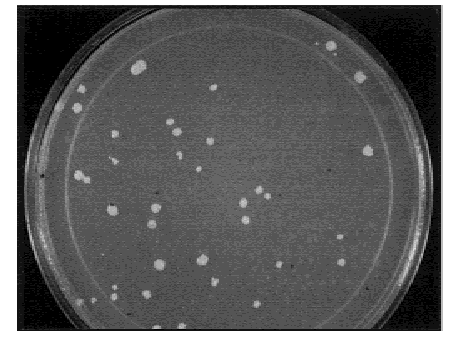

$\mathrm{SC}+3 \mathrm{AT} \# 1$

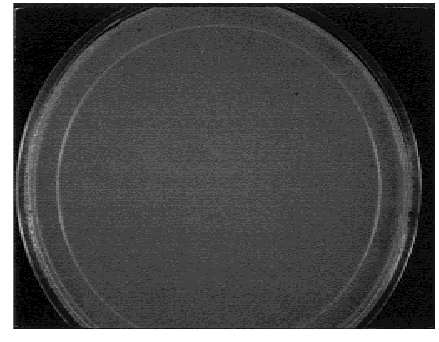

$\mathrm{SC}+3 \mathrm{AT} \# 2$

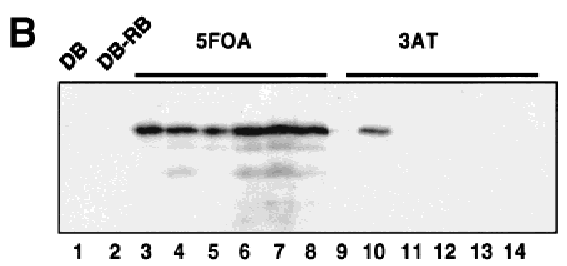

Figure 3 Preclearing of PCR-generated self-activator baits. (A) A PCRgenerated lin-5 sequence was transformed by Gap repair into MaV103 cells. The $\mathrm{Leu}^{+}$transformants were treated as described in the legend to Fig. 1. The plates shown here correspond to the Sc-Leu, Sc+3AT\#1, and $\mathrm{SC}+3 \mathrm{AT} \# 2$ plates indicated in red in Fig. 1. (B) Western-blot analysis of extracts from yeast cells expressing self-activator (lanes 9-14) and nonself-activator (lanes 3-8) DB-LIN-5 baits. A mix of three anti-LIN-5 monoclonal antibodies that recognize epitopes in the LIN-5 carboxyl terminus were used. (Lanes 1,2) Extracts from negative control cells expressing $\mathrm{DB}$ alone and $\mathrm{DB}-\mathrm{RB}$, respectively ( $\mathrm{RB}$ corresponds to the pocket domain of the human retinoblastoma protein); (lanes 3,9) extracts from pools of colonies; (lanes 4-8, 10-14) extracts from individual colonies.

phenotype, suggesting that a subpopulation of DBLIN-5 behaves as spurious self-activator baits (Fig. 3A, left, bottom).

The genetic selections used here to preclear the self-activator baits were identical to those described above except that only one round of 5FOA selection was sufficient to eliminate all self-activator-expressing colonies (not shown). The preclearing ratio compared with our control was satisfactory, and this is exemplified be the fact that no 3AT-growing colonies could be observed after the recovery on Sc-Leu plates (Fig. 3A, right, bottom). 
To address the efficiency of two-hybrid selections subsequent to the preclearing step, a screen was performed with the precleared DB-LIN-5 bait and the data obtained were compared with those from $\sim 100$ screens performed without any preclearing step. The LIN-5 interactors identified are still being characterized and will be described elsewhere (M. Lorson, A.J.M. Walhout, M. Vidal, and S. van den Heuvel, in prep.). However, for the purpose of this experiment, the number of different potential interactors found at least twice can be a good indicator of the quality of a screen. Five potential interactors were found for LIN-5, which is in good agreement with the average number of interactors found in the 100 screens mentioned above (M. Vidal, in prep.). Thus preclearing can be applied to DB-X baits that give rise to spurious self activators without significantly affecting the read out of twohybrid screens.

Finally, we were intrigued by the nature of the molecular events that could give rise to spurious selfactivator baits after PCR amplification. As recognized previously, PCR amplification can be very mutagenic (Mulhard et al. 1992). At least three classes of mutations can be envisioned, including missense mutations that lead to single amino acid changes and nonsense or frame-shift mutations that result in a truncated protein. Interestingly, it has been observed that truncated versions of several proteins exhibit transcriptional activation, whereas the corresponding full length does not. Different folding properties of truncated and fulllength proteins are sometimes argued to explain this phenomenon. Thus, we decided to test whether the PCR-induced DB-LIN-5-mutated self activators correspond to truncated versions of the fusion protein. An immunoblot was performed on yeast extracts prepared from both 5FOA-resistant (non-self activators) and 3AT-resistant (self activators) colonies, with a mixture of three anti-LIN-5 monoclonal antibodies that recognize the LIN-5 carboxyl terminus (M. Lorson, H. R. Horvitz, and S. van den Heuvel, in prep.) (Fig. 3B). As expected, five of five 5FOA-resistant colonies expressed full-length DB-LIN-5 protein. However, four of five 3AT-resistant clones did not. The absence of a LIN-5specific signal in most extracts derived from 3ATresistant clones is consistent with the idea that PCRinduced mutations can create stop codons or frameshift mutations leading to truncated products. It is possible that spurious activation domains might be accessible to the yeast transcriptional machinery only in the context of truncated rather than full-length proteins.

\section{Conclusions}

Self-activator baits cannot be used in conventional forward two-hybrid selections because they do not depend on any interaction to elevate the expression of the reporter genes. The percentage of such sequences in random cDNA (or genomic) DB-X libraries or in PCR-generated DB-X baits can be higher than the frequency of genuine interactors by several orders of magnitude. Thus, an efficient method is needed to eliminate them prior to performing a forward two-hybrid selection for potential AD-Y interactors.

We have shown here that negative-selectiongrowth phenotypes can be used to eliminate such self activators from pools of DB-X baits, either present naturally in cDNA libraries, or generated by PCR.

We note that relevant proteins that exhibit selfactivation properties in this assay, and thus would be eliminated as DB-X bait fusions, will be tolerated when expressed as AD-Y fusions. Thus, they will not necessarily be precluded from large-scale interaction screens. In theory however, one might imagine situations in which both interacting proteins exhibit self-activation properties. For these cases, an alternative configuration of the two-hybrid system that makes use of Pol III transcriptional regulation could be used (Marsolier et al. 1997).

We envision that the feasibility and quality of protein-interaction mapping projects will be greatly improved with the genetic selection against self activators described here.

\section{METHODS}

Yeast Manipulations and DB- and AD-cDNA Libraries The yeast strains MaV103 and MaV203 have been described previously (Vidal et al. 1996a). All information concerning yeast manipulations such as medium, incubation time, and replica plating can be found in Vidal (1997). The 13.5 mouse embryonic AD-cDNA library used in Figure 2 was described in Hu et al. (1997). The 13.5 mouse embryonic DB-cDNA library used in Figure 2 is a kind gift from M. Heymans and R. Bernards (N.K.I., Amsterdam, The Netherlands). It was generated by cloning into pPC97 (Vidal 1997) the inserts from a preexisting AD-cDNA library described in Chevray and Nathans (1992) (details will be published later). The worm AD-cDNA library used in Figure 3 will be described in detail elsewhere. PCR reactions directly from yeast cells were performed essentially as described (Wang et al. 1996). Automated sequencing was performed as described in the Perkin Elmer ABI protocol.

\section{PCR/Gap Repair}

PCR reactions $(50 \mu \mathrm{l})$ were assembled on ice and contained 60 mM Tris- $\mathrm{SO}_{4}$ at $\mathrm{pH} 9.1,18 \mathrm{~mm}\left(\mathrm{NH}_{4}\right)_{2} \mathrm{SO}_{4}, 2 \mathrm{~mm} \mathrm{MgSO}_{4}, 50 \mu \mathrm{g}$ of BSA, $200 \mu \mathrm{M}$ dNTP, $0.5 \mu \mathrm{M}$ of each primer, $100 \mathrm{ng}$ of CMVlin-5 (M. Lorson and S. van den Heuvel, in prep.), and 0.25 units of Elongase Taq polymerase (Life Technologies Inc.). The i $i$ - 5 forward primer was 5 '-TAGTAACAAAGGTCAAAGACA G G T T GACTGTA T CGT CGAGGA G C G T G A GCACATCAGTTGTG-3' (the second codon of the lin-5 ORF is underlined). The lin-5 reverse primer was $5^{\prime}$-GCCGTTA CT TACTTAGAGCTCGACG TCTTACT TACTTAGCTTACTGCTTTTTGCTCGAAAA-3' (the lin-5 stopcodon is underlined). Sequences identical to the pPC97 polylinker sequences and required for Gap repair are indicated in bold. The lin-5 sequence was amplified in a reac- 
tion consisting of 4 cycles with an annealing temperature of $56^{\circ} \mathrm{C}$ and 11 cycles with a temperature of $66^{\circ} \mathrm{C}$. For Gap repair, MaV103 cells were transformed with 25 ng of pPC97 digested with SalI and BglII, along with $5 \mu$ of PCR product. Colonies were grown on plates SC-Leu to select for pPC97derived plasmids. Controls included no DNA, linearized vector alone, PCR product alone, and circular pPC97. An increase in the number of colonies of at least 20 -fold was observed between linearized vector alone and vector + PCR product.

\section{Western Blot Analysis}

The Western blot analysis was performed as described (Vidal 1997). The anti-LIN-5 monoclonal antibodies were raised against a carboxy-terminal domain of the protein (M. Lorson, H. R. Horvitz, and S. van den Heuvel, in prep.).

\section{ACKNOWLEDGMENTS}

We thank our friends and colleagues J. Dekker, L. Matthews and $\mathrm{M}$. Polymenis for careful reading of the manuscript, $\mathrm{M}$. Fitzgerald for help with the sequencing of the Globinencoding clones, S. van den Heuvel and M. Lorson for their gift of unpublished sequences and antibodies, and their help with questions relating to C. elegans biology, M. Heymans and R. Bernards for the DB-cDNA 13.5-day embryonic library, and E. Harlow for his support at an early phase of this project. The work described here was funded by Grants nos. IRG-173H from the American Cancer Society and 1 RO1 HG01715 A1 from the National Human Genome Research Institute to M.V.

The publication costs of this article were defrayed in part by payment of page charges. This article must therefore be hereby marked "advertisement" in accordance with 18 USC section 1734 solely to indicate this fact.

\section{REFERENCES}

Bartel, P.L., J.A. Roecklein, D. SenGupta, and S. Fields. 1996. A protein linkage map of Escherichia coli bacteriophage T7. Nat. Genet. 12: 72-77.

Blattner, F.R., G.R. Plunkett, C.A. Bloch, N.T. Perna, V. Burland, M. Riley, J. ColladoVides, J.D. Glasner, C.K. Rode, G.F. Mayhew et al. 1997. The complete genome sequence of Escherichia coli K-12. Science 277: 1453-1474.

Boeke, J.D., F. LaCroute, and G.R. Fink. 1984. A positive selection for mutants lacking orotidine-5'-phosphate decarboxylase activity in yeast: 5-fluoro-orotic acid resistance. Mol. Gen. Genet. 197: 345-346.

C. elegans Sequencing Consortium. 1998. Genome sequence of the nematode C. elegans: A platform for investigating biology. Science 282: 2012-2018.

Chevray, P. and D. Nathans. 1992. Protein interaction cloning in yeast: Identification of mammalian proteins that react with the leucine zipper of Jun. Proc. Natl. Acad. Sci. 89: 5789-5793.

Choi, K.Y., B. Satterberg, D.M. Lyons, and E.A. Elion. 1994. Ste5 tethers multiple protein kinases in the MAP kinase cascade required for mating in S. cerevisiae. Cell 78: 499-512.

Du, W., M. Vidal, J.-E. Xie, and N. Dyson. 1996. RBF, a novel RB-related gene that regulates E2F activity and interacts with cyclin E in Drosophila. Genes \& Dev. 10: 1206-1218.

Durfee, T., K. Becherer, P.-L. Chen, S.-H. Yeh, Y. Yang, A.E. Kilburn, W.-H. Lee, and S.J. Elledge. 1993. The retinoblastoma protein associates with the protein phosphatase type 1 catalytic subunit. Genes \& Dev. 7: 555-569.

Evangelista, C., D. Lockshon, and S. Fields. 1997. The yeast two-hybrid system: Prospects for protein linkage maps. Trends Cell Biol. 6: 196-199.

Fields, S. 1997. The future is function. Nat. Genet. 15: 325-327.

Fields, S. and O. Song. 1989. A novel genetic system to detect protein-protein interactions. Nature 340: 245-246.

Finley, R.L., Jr. and R. Brent. 1994. Interaction mating reveals binary and ternary connections between Drosophila cell cycle regulators. Proc. Natl. Acad. Sci. 91: 12980-12984.

Flores, A., J. Briand, O. Gadal, J. Andrau, L. Rubbi, V. Van Mullem, C. Boschiero, M. Goussot, C. Marck, C. Carles et al. 1999. A protein-protein interaction map of yeast RNA polymerase III. Proc. Natl. Acad. Sci. 96: 7815-7820.

Fromont-Racine, M., J.C. Rain, and P. Legrain. 1997. Toward a functional analysis of the yeast genome through exhaustive two-hybrid screens. Nat. Genet. 16: 277-282.

Goffeau, A., R. Aert, M.L. Agostini-Carbone, A. Ahmed, M. Aigle, L. Alberghina, K. Albermann, M. Albers, M. Aldea, D. Alexandraki et al. 1997. The yeast genome directory. Nature (suppl.) 387: 1-105.

Guyer, M.S. and F.S. Collins. 1995. How is the Human Genome Project doing, and what have we learned so far? Proc. Natl. Acad. Sci. 92: 10841-10848.

Hu, G., S. Zhang, M. Vidal, J. Labaer, T. Xu, and E.R. Fearon. 1997. Mammalian homologs of seven in absentia regulate DCC via the ubiquitin-proteasome pathway. Genes \& Dev. 11: 2701-2714.

Hudson, J.R., E.P. Dawson, K.L. Rushing, C.H. Jackson, D. Lockshon, D. Conover, C. Lanciault, J.R. Harris, S.J. Simmons, R. Rothstein, and S. Fields. 1997. The complete set of predicted genes from Saccharomyces cerevisiae in a readily usable form. Genome Res. 7: 1169-1173.

Koleske, A.J. and R.A. Young. 1994. An RNA polymerase II holoenzyme responsive to activators. Nature 368: 466-469.

Lander, E.S. 1996. The new genomics: Global views of biology. Science 274: 536-539.

Ma, J., and M. Ptashne 1987. A new class of yeast transcriptional activators. Cell 51: 113-119.

Marsolier, M.C., M.N. Prioleau, and A. Sentenac. 1997. A RNA polymerase III-based two-hybrid system to study RNA polymerase II transcriptional regulators. J. Mol. Biol. 268: 243-249.

Mulhard, D., R. Hunter, and R. Parker. 1992. A rapid method for localized mutagenesis of yeast genes. Yeast 8: 79-82.

Schena, M., D. Shalon, R.W. Davis, and P.O. Brown. 1995. Quantitative monitoring of gene expression patterns with a complementary DNA microarray. Science 270: 467-470.

Smith, V., K.N. Chou, D. Lashkari, D. Botstein, and P.O. Brown. 1996. Functional analysis of the genes of yeast chromosome V by genetic footprinting. Science 274: 2069-2074.

Soares, M.B., M.F. Bonaldo, P. Jelene, L. Su, L. Lawton, and A. Efstratiadis. 1994. Construction and characterization of a normalized cDNA library. Proc. Natl. Acad. Sci. 91: 9228-9232.

Stephens, R.S., S. Kalman, C. Lammel, J. Fan, R. Marathe, L. Aravind, W. Mitchell, L. Olinger, R.L. Tatusov, Q. Zhao et al. 1998. Genome sequence of an obligate intracellular pathogen of humans: Chlamydia trachomatis. Science 282:754-759.

Vidal, M. 1997. The reverse two-hybrid system. In The yeast two-hybrid system (ed. P. Bartel and S. Fields) pp. 109-147 Oxford University Press, Oxford, UK.

Vidal, M. and H. Endoh 1999. Prospects for drug screening using the reverse two-hybrid system. Trends in Biotechnol. 17: 374-381.

Vidal, M. and P. Legrain 1999. Yeast forward and reverse " $n$ "-hybrid systems. Nucleic Acids Res. 27: 919-929.

Vidal, M., R. Brachmann, A. Fattaey, E. Harlow, and J.D. Boeke. 1996a. Reverse two-hybrid and one-hybrid systems to detect dissocation of protein-protein and DNA-protein interactions. Proc. Natl. Acad. Sci. 93: 10315-10320.

Vidal, M., P. Braun, E. Chen, J.D. Boeke, and E. Harlow. 1996b. Genetic characterization of a mammalian protein-protein interaction using a reverse two-hybrid system. Proc. Natl. Acad. Sci. 93: 10321-10326.

Walhout, A.J.M., H. Endoh, N. Thierry-Mieg, W. Wong, and M. Vidal. 1998. A model of elegance. Am. J. Hum. Genet. 63: 955-961.

Wang, H., E. Kohalmi, and A.J. Cutler. 1996. An improved method for polymerase chain reaction using whole yeast cells. Anal. Biochem. 237: 145-146.

Received May 12, 1999; Accepted in revised form September 16, 1999.

\section{Genome Research}




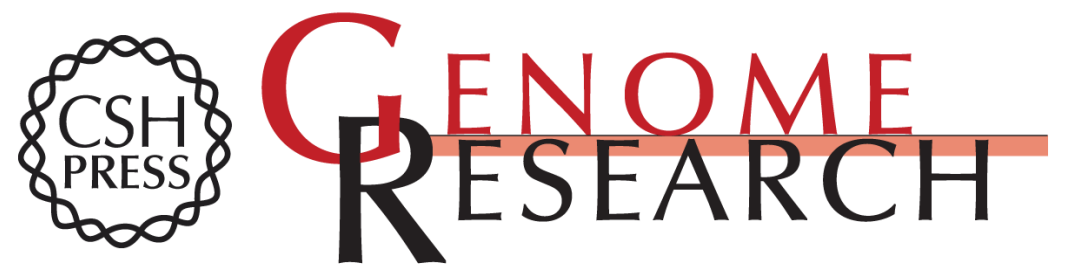

\section{A Genetic Strategy to Eliminate Self-Activator Baits Prior to High-Throughput Yeast Two-Hybrid Screens}

Albertha J.M. Walhout and Marc Vidal

Genome Res. 1999 9: 1128-1134

Access the most recent version at doi:10.1101/gr.9.11.1128

References This article cites 31 articles, 17 of which can be accessed free at:

http://genome.cshlp.org/content/9/11/1128.full.html\#ref-list-1

\section{License}

Email Alerting Receive free email alerts when new articles cite this article - sign up in the box at the Service top right corner of the article or click here.

\section{Affordable, Accurate Sequencing.}

